\title{
Mecânica Clássica - Pontos de equilíbrio e pontos de retorno
}

\author{
Mario Cezar Bertin ${ }^{1}$ \\ ${ }^{1}$ Affiliation not available
}

November 2, 2020

\begin{abstract}
Nesta seção, estudaremos pontos de equiíbrio e pontos de retorno de potenciais unidimensionais. Pontos de equilíbrio estão relacionados a estados estáticos estáves e instáveis de um sistema, através dos pontos críticos do potencial, de primeira derivada nula. Estudaremos, também, pontos de retorno, que são pontos de energia cinética nula de um sistema.
\end{abstract}

\section{Introdução}

Em seções passadas, vimos que um sistema unidimensional sujeito à conservação de sua energia mecânica fornece formas de se calcular a curva, ou função horária, realizada pelo sistema. No caso de uma partícula sujeita a um potencial unidimensional, se sua energia

$$
E=\frac{1}{2} m \dot{x}^{2}+V(x)
$$

se conserva, temos

$$
\frac{d E}{d t}=0 \quad \Longrightarrow \quad m \dot{x} \ddot{x}+\frac{d V}{d x} \dot{x}=0
$$

leva à equação de movimento

$$
m \ddot{x}=-\frac{d V}{d x}
$$

que é nada menos que a segunda lei de Newton aplicada ao sistema. Por outro lado, se $E$ é uma constante de movimento, a eq. (??) resulta na quadratura

$$
\Delta t= \pm \sqrt{\frac{m}{2}} \int_{x_{0}}^{x} \frac{d x^{\prime}}{\sqrt{E-V\left(x^{\prime}\right)}}
$$

Encontrar a curva solução do sistema implica em resolver (??) ou a quadratura (??), a critério.

Contudo, a quadratura nos fonece um conjunto de informações que a equação de movimento, a princípio, não fornece. Por exemplo, o argumento da raiz do denominador, dado pela expressão $E-V(x)$, deve ser sempre maior que zero, sob pena de o denominador da integral ser nulo ou, até mesmo, um número imaginário. Isto implica, em qualquer ponto da trajetória, em que a energia deve ser sempre maior que o valor do potencial naquele ponto. Portanto, uma trajetória com pontos nos quais $E<V$ é proibida. Por outro lado, pontos em que $E=V$ são pontos limites do movimento da partícula, em que a energia cinética $K$ é nula e, portanto, a velocidade é nula. Vamos ver como essas informações são úteis na descrição dos sistemas mecânicos. 


\section{Pontos de equilíbrio}

Vamos supor um sistema unidimensonal de potencial $V(x)$, dependente apenas da posição. Na figura 1 , temos um exemplo de gráfico da função

$$
V(x)=\frac{1}{4} x^{2},
$$

que é um exemplo particular do potencial do oscilador harmônico simples.

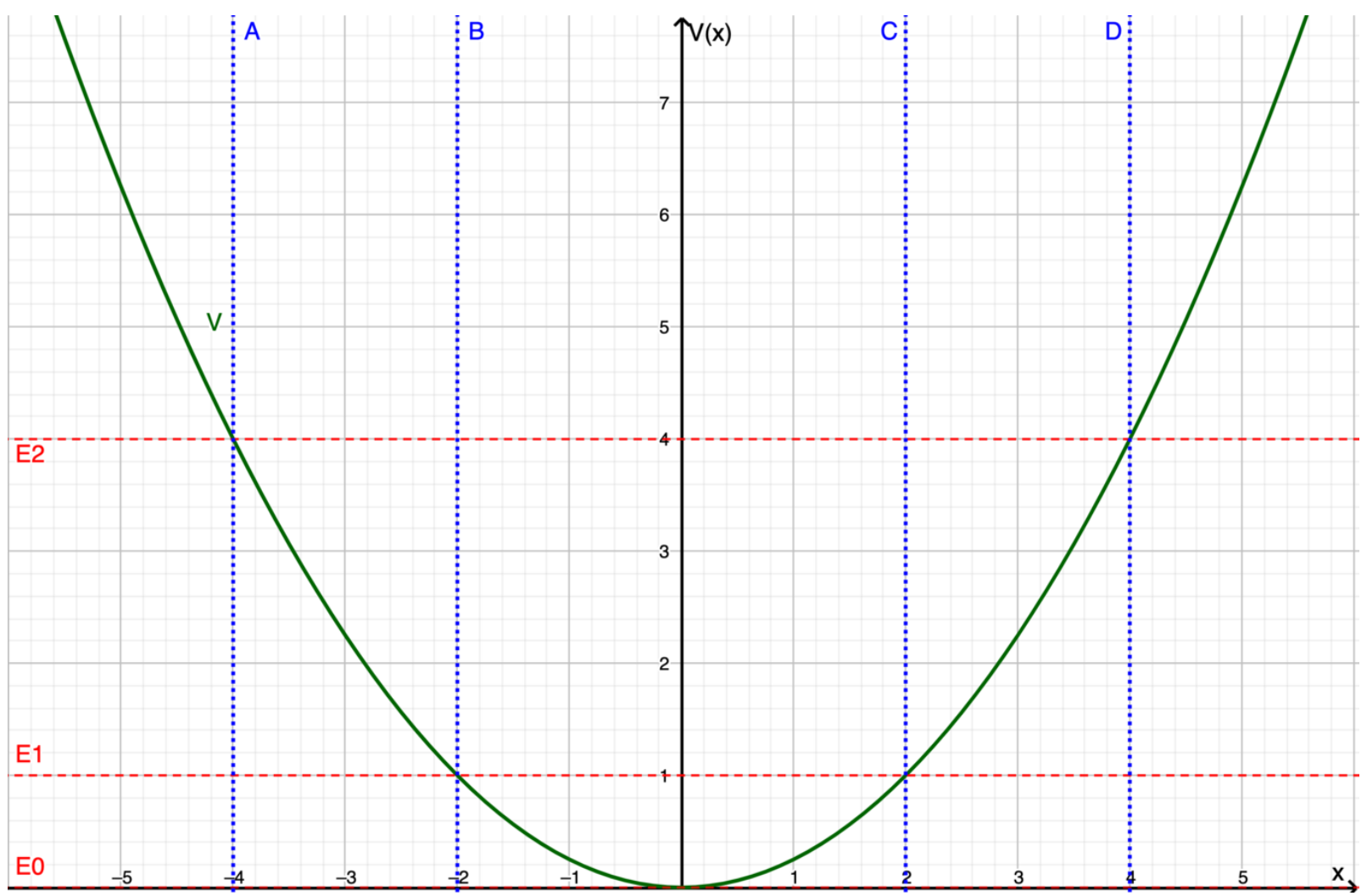

Figure 1: Gráfico do potencial do oscilador harmônico simples.

No gráfico acima, temos também representados três valores de energia mecânica, $E_{0}=0, E_{1}=1$ e $E_{2}=4$, por enquanto, vamos ignorar o sistema de unidades utilizado. Como a energia mecânica é constante, seu gráfico é uma reta horizontal com valor igual ao valor da energia. Cada gráfico de energia intersecta o gráfico do potencial em dois pontos: a energia $E_{0}$ é igual ao potencial apenas no ponto $x=0$; a energia $E_{1}$ é igual ao potencial nos pontos $x= \pm 2$, representados pelas retas $B$ e $C$ em azul; por fim, a energia $E_{2}$ é igual ao potencial em $x= \pm 4$, como mostram as retas $A$ e $D$.

Especialmente no ponto $x=0$, o potencial tem seu valor mínimo global $V=0$, de modo que nenhum ponto do domínio tem valor de potencial menor, ou mesmo igual. Dizemos que $x=0$ é um mínimo global deste potencial. Este é, também, um ponto de equilíbrio estável, o que significa que qualquer partícula colocada neste ponto tem força instantânea igual a zero, mas se perturbada, tende a voltar a esta posição em razão de uma força restauradora. Isto pode ser visto através da função da força do oscilador:

$$
F=-\frac{d V}{d x}=-\frac{1}{2} x
$$

que é negativa para $x>0$, positiva para $x<0$ e nula para $x=0$. 
Usando esta ideia, definimos os pontos de equilíbrio:

Um ponto de equilíbrio, ou ponto crítico de um potencial, consiste em um ponto da trajetória no qual a força exercida sobre o sistema é nula.

Como a força é derivada do potencial pela relação $\mathbf{F}=-\nabla V$, o que resulta em $F=-d V / d x$ em uma dimensão, pontos de equilíbrio são encontrados nos pontos nulos da primeira derivada do potencial unidimensional e, portanto, são pontos críticos matemáticos da função $V(x)$. Portanto,

Pontos de equilíbrio são raízes da equação

$$
\frac{d V(x)}{d x}=0
$$

\section{O teorema de Taylor}

Para compreender os tipos de pontos de equilíbrio, precisamos recorrer ao teorema de Taylor. O teorema expressa que toda função de uma variável diferenciável $n$ vezes em um ponto $x=x^{\prime}$ pode ser descrita pela série

$$
\begin{gathered}
f(x)=a m p ; f\left(x^{\prime}\right)+\left.\frac{1}{1 !} \frac{d f}{d x}\right|_{x=x^{\prime}}\left(x-x^{\prime}\right)+\left.\frac{1}{2 !} \frac{d^{2} f}{d x^{2}}\right|_{x=x^{\prime}}\left(x-x^{\prime}\right)^{2}+ \\
a m p ;+\cdots+\left.\frac{1}{n !} \frac{d^{n} f}{d x^{n}}\right|_{x=x^{\prime}}\left(x-x^{\prime}\right)^{n}+R,
\end{gathered}
$$

o que também pode ser escrito por

$$
f(x)=\left.\sum_{i=0}^{n} \frac{1}{i !} \frac{d^{i} f}{d x^{i}}\right|_{x=x^{\prime}}\left(x-x^{\prime}\right)^{i}+R,
$$

em que $R$ é denominada resto. Se

$$
\lim _{x \rightarrow x^{\prime}} R=0,
$$

dizemos que $f$ é uma função analítica em $x=x^{\prime}$.

A prova deste toerema foge ao escopo dessas aulas, mas podemos utilizá-lo para analisar o que ocorre com um potencial que pode ser aproximado pela série de Taylor (??) na forma

$$
V(x) \approx V\left(x^{\prime}\right)+\left.\frac{1}{1 !} \frac{d V}{d x}\right|_{x=x^{\prime}}\left(x-x^{\prime}\right)+\left.\frac{1}{2 !} \frac{d^{2} V}{d x^{2}}\right|_{x=x^{\prime}}\left(x-x^{\prime}\right)^{2} .
$$

para $x-x^{\prime}$ suficientemente pequeno. Aqui, o resto é considerado muito pequeno para constribuir para o valor de $V$, portanto, a expressão (??) é uma aproximação adequada para $V(x)$ desde que $x$ esteja suficientemente próximo de $x^{\prime}$.

\section{Tipos de equilíbrio}

Agora, vamos supor que $x=x^{\prime}$ seja um ponto de equilíbrio de $V$. Neste caso, definindo-se $\Delta V \equiv V(x)-$ $V\left(x^{\prime}\right)$, temos

$$
\left.\Delta V \approx \frac{1}{2 !} \frac{d^{2} V}{d x^{2}}\right|_{x=x^{\prime}}\left(x-x^{\prime}\right)^{2}
$$

visto que $d V / d x=0$ para $x=x^{\prime}$, como condição de criticidade. Agora, temos dois casos:

1. A segunda derivada de $V$ em $x=x^{\prime}$ é positiva: Neste caso, a diferença de potencial $\Delta V$ é positiva, o que significa $V(x)>V\left(x^{\prime}\right)$. Neste caso, $x=x^{\prime}$ é um ponto de mínimo local do potencial. 
Vamos verificar o que ocorre com a força nesta aproximação. Note, primeiro, que

$$
\frac{d}{d x} \Delta V=\left.\frac{d V}{d x} \approx \frac{d^{2} V}{d x^{2}}\right|_{x=x^{\prime}}\left(x-x^{\prime}\right)=F(x) .
$$

Assim, se $x=x^{\prime}$ é um ponto de mínimo, $F(x)>0$ para $x<x^{\prime}$ e $F(x)<0$ para $x>x^{\prime}$. Esta é uma força restauradora. Neste caso, dizemos que

Um ponto de mínimo local de um potencial é um ponto de equilíbrio estável.

Como comentamos anteriormente, um ponto de equilíbrio estável é caracterizado pela existência de forças restauradoras contra qualquer ação que retire o sistema do equilíbrio. No caso do oscilador harmônico simples da figura 1 , o ponto $x=0$ é um ponto de equilíbrio estável. De fato, o potencial do oscilador já possui forma quadrática típica da expressão (??), portanto, a série de Taylor do oscilador é exata, tendo resto zero.

2. A segunda derivada de $V$ em $x=x^{\prime}$ é negativa: Neste caso, a diferença de potencial $\Delta V$ é negativa, o que significa $V(x)<V\left(x^{\prime}\right)$. Neste caso, $x=x^{\prime}$ é um ponto de máximo local do potencial.

No caso 2, observando-se a equação (??), temos que a força é positiva para $x>x^{\prime}$ e negativa para $x<x^{\prime}$, portanto, esta é uma força de escape, pois seu módulo aumenta com o aumento da distância absoluta $\left|x-x^{\prime}\right|$. Por esta razão,

Um ponto de máximo local de um potencial é um ponto de equilíbrio instável.

O oscilador harmônico simples não possui pontos de equilíbrio instáveis. Mas um potencial do tipo $V=\cos x$, por exemplo, possui infinitos desses pontos para $x \in \mathbb{R}$. Em contrapartida o mesmo potencial possui também infinitos pontos de equilíbrio estáveis.

Há, ainda, o caso em que a segunda derivada do potencial é nula. Se isso ocorrer, a aproximação (??) não é adequada para $x \rightarrow x^{\prime}$. Assim, devemos recorrer a termos de ordem superior da série de Taylor, como por exemplo o termo

$$
\left.\Delta V \approx \frac{1}{3 !} \frac{d^{3} V}{d x^{3}}\right|_{x=x^{\prime}}\left(x-x^{\prime}\right)^{3},
$$

que é representado como exemplo pelo gráfico abaixo: 


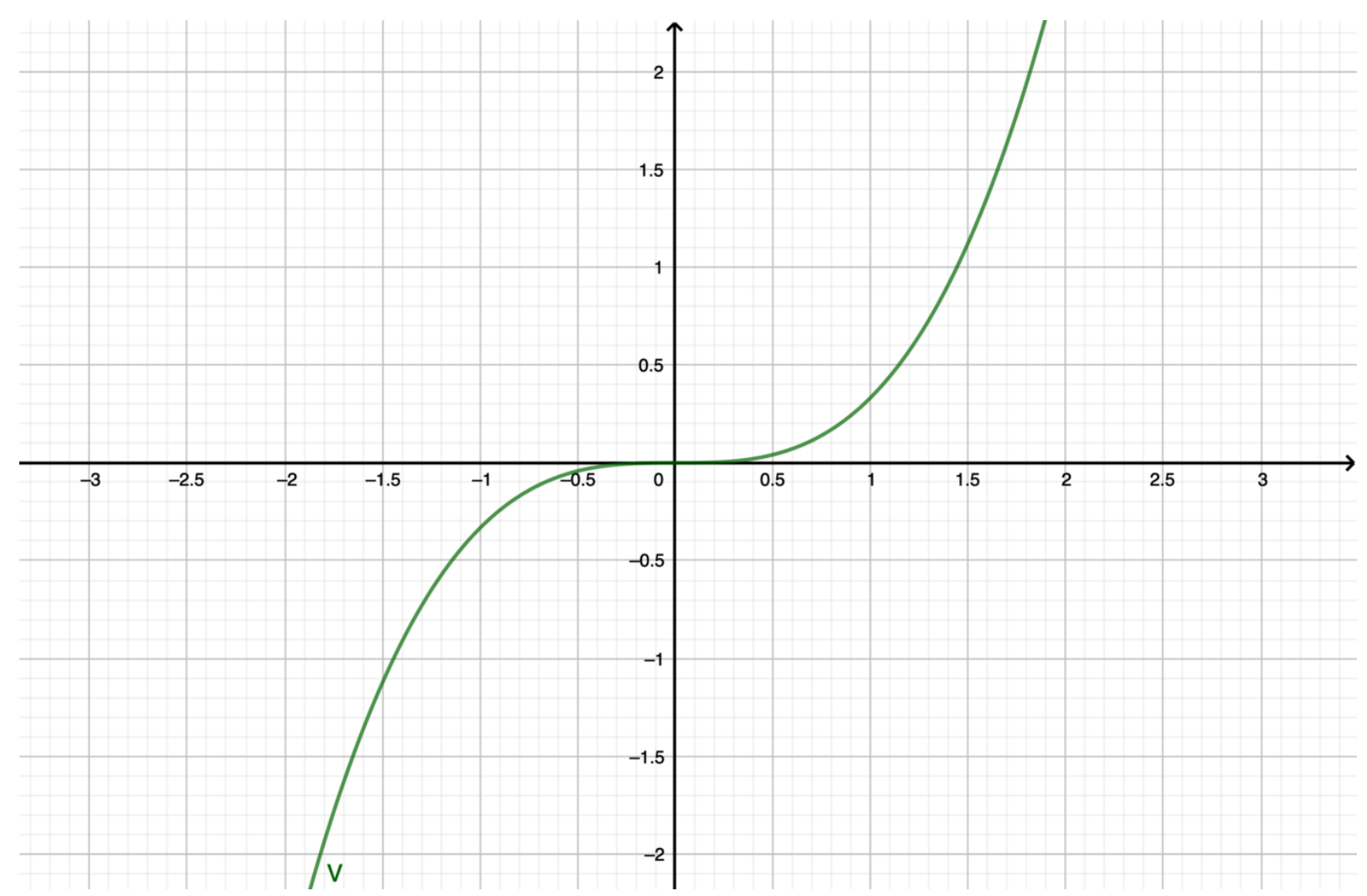

Figure 2: Gráfico da função $x^{3} / 3$.

No gráfico 2, o ponto $x=0$ não é um ponto de máximo ou de mínimo. Mas é claramente um ponto de equilíbrio instável, visto que qualquer perturbação sobre a partícula vai resultar, eventualmente, em uma força de escape.

Caso a terceira derivada seja nula, devemos ir à quarta ordem de aproximação, e assim por diante. Se todas as derivadas ao redor de $x=x^{\prime}$ forem nulas, o potencial é constante e temos, por consequência, uma partícula livre. Uma partícula livre está sempre em equilíbrio.

\section{Pontos proibidos e pontos de retorno}

A função potencial de um sistema também define regiões proibidas no domínio do sistema. São as regiões nas quais a energia cinética possui valores negativos no caso de problemas unidimensionais.

Podemos ver a proibição de funções horárias com energia cinética negativa mais facilmente na integral de quadratura

$$
t-t_{0}=\sqrt{\frac{m}{2}} \int_{x_{0}}^{x} \frac{d x^{\prime}}{\sqrt{E-V\left(x^{\prime}\right)}} .
$$

O denominador no argumento da integral contém uma raiz quadrada, que deve ser real e diferente de zero. Portanto, a condição

$$
E-V(x)>0
$$

é condição necessária para a existência de curvas físicas que representem o movimento do sistema. 
O caso do oscilador harmônico simples, mais uma vez, fornece um exemplo clássico. Na figura, apresentam-se linhas de energia constante, através das funções $E_{0}=0, E_{1}=1$ e $E_{2}=4$. Essas retas intersectam o potencial nos pontos $x=0, x= \pm 2$ e $x= \pm 4$ respectivamente. Esses pontos marcam as ocasiões nas quais a energia cinética é nula e $E=V$, caso limite da condição (??). Energia cinética nula implica em módulo da velocidade nula. Esses pontos são chamados pontos de retorno do potencial.

Vamos começar com o sistema descrito pelo gráfico ?? com $E=E_{0}=0$. Como a partícula é proibida de frequentar qualquer subconjunto do domínio em que $E<V$, o único ponto permitido é a posição $x=0$, que é precisamente o mínimo do potencial. Este é o único ponto de equilíbrio, e é um ponto de equilíbrio estável, como já vimos. Portanto, a única configuração possível para um oscilador com energia zero é o repouso no ponto de equilíbrio. Não são permitidas energias negativas neste sistema, pois o zero é o menor valor possível para o potencial.

Podemos aumentar a energia do sistema empregando um impulso com certa velocidade inicial, para imprimir à partícula certa energia cinética. Como a energia mecânica total se conserva, esta energia é precisamente a energia cinética empregada no ponto $x=0$. Vamos supor que, no caso do sistema do gráfico 1, esta energia seja $E=E_{1}=1$. Neste caso, os pontos proibidos serão todos aqueles à esquerda de $x=-2$ e aqueles à direita de $x=2$, ou seja, os pontos permitidos são aqueles $x \in(-2,2)$. Com um pouco mais de impulso, podemos imprimir ao sistema uma energia maior, digamos $E=E_{2}=4$. Neste caso, o intervalo permitido é dado por $x \in(-4,4)$. Em qualquer desses casos, se $E>V_{\text {min }}$, a partícula percorre sua trajetória em um intervalo de valores permitido pela limitação da sua energia cinética. Ao encontrar um ponto de retorno, a partícula atinge um ponto de velocidade nula e retoma o movimento em sentido contrário. Sempre que um sistema possui dois pontos de retorno fixos em sua trajetória, o movimento é denominado oscilatório.

Portanto, os pontos de retorno do oscilador harmônico simples são raízes da equação

$$
E-V(x)=0 \quad \Longrightarrow \quad \frac{1}{2} m \omega^{2} x^{2}=E
$$

que resulta em

$$
x^{2}=\frac{2 E}{m \omega^{2}} \quad \Longrightarrow \quad x_{ \pm}= \pm \sqrt{\frac{2 E}{m \omega^{2}}} .
$$

Portanto, o potencial quadrático possui dois pontos de retorno, que denominamos $x_{ \pm}$.

\section{O Potencial de Lennard-Jones}

Um exemplo mais interessante vem a ser o potencial de Lennard-Jones. Este é um potencial efetivo (falaremos disso mais adiante), o que significa que ele modela uma interação unidimensional em um sistema tridimensional. O sistema em questão é uma molécula diatômica neutra. Este potencial tem a forma

$$
V(r)=\frac{\alpha}{r^{12}}-\frac{\beta}{r^{6}},
$$

com constantes $\alpha$ e $\beta$ reais positivas. A coordenada $r$ representa a distância de separação entre os núcleos dos átomos que formam a molécula. Uma molécula diatômica é um sistema em três dimensões, que possui seis graus de liberdade, o que pode ser um sistema bastante complicado. Contudo, a aproximação para um potencial do tipo Lennard-Jones é bastante utilizada. Um exemplo de gráfico deste potencial está na figura 3. 


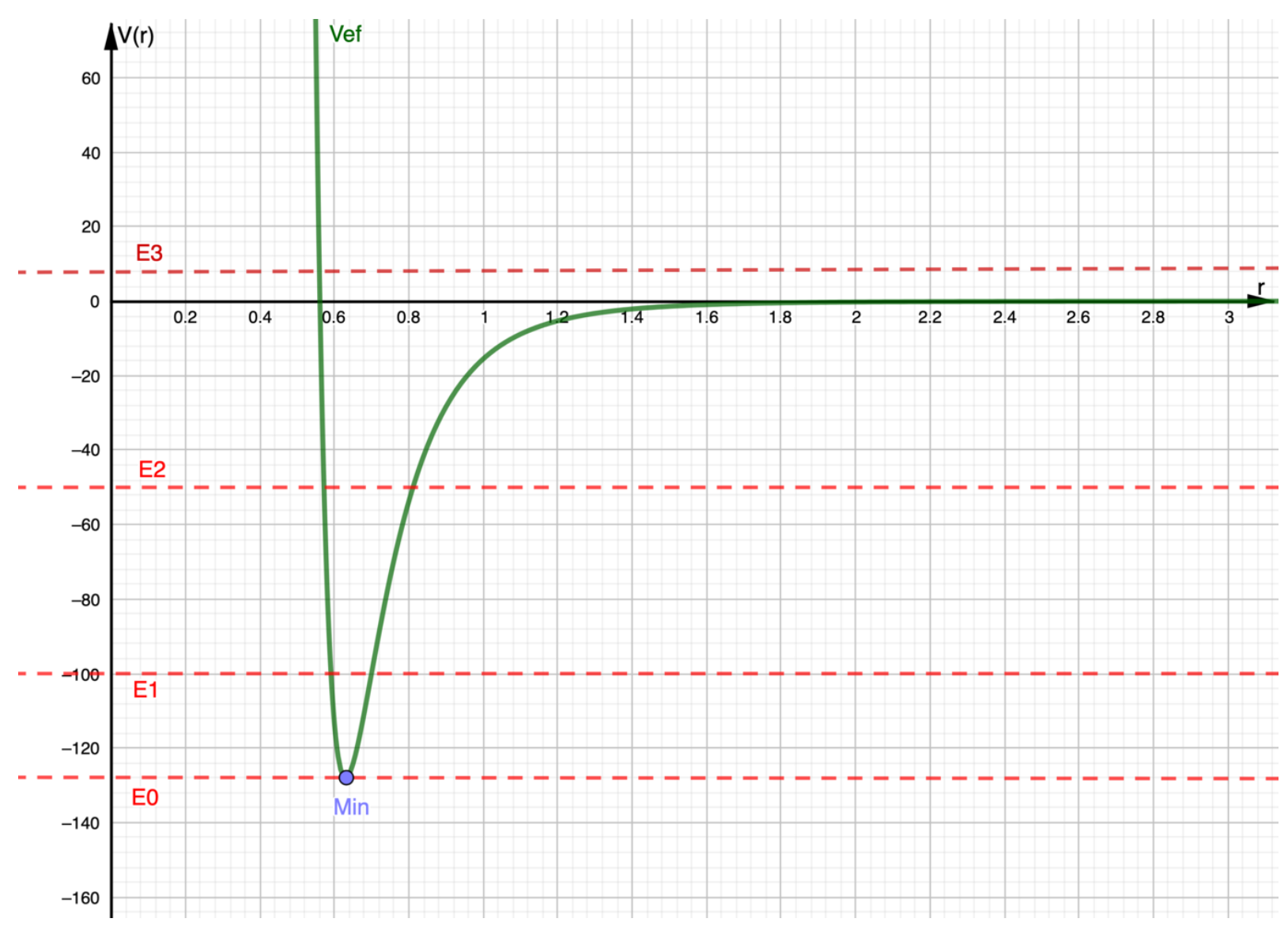

Figure 3: Gráfico do Potencial de Lennard-Jones.

No gráfico 3, vemos que o potencial de Lennard-Jones possui apenas um ponto de mínimo, de equilíbrio estável global, aproximadamente em $r=0,62$. Também possui energia mínima em $E=E_{0} \approx-124$, e para $E_{0}<E<0$, existem dois pontos de retorno. Para energias positivas, o potencial tem apenas um ponto de retorno.

Esses valores são calculados através do próprio potencial. No caso do ponto de equilíbrio, temos

$$
\frac{d V}{d r}=-12 \frac{\alpha}{r^{13}}+6 \frac{\beta}{r^{7}}=\frac{6}{r^{7}}\left[\beta-2 \frac{\alpha}{r^{6}}\right],
$$

que tem raizes definidas pela equação

$$
\beta-2 \frac{\alpha}{r^{6}}=0 \quad \Longrightarrow \quad r_{0}=\left[\frac{2 \alpha}{\beta}\right]^{1 / 6}
$$

ou seja, apenas uma raiz positiva $r_{0}$.

A segunda derivada do potencial é dada por

$$
\frac{d^{2} V}{d r^{2}}=156 \frac{\alpha}{r^{14}}-42 \frac{\beta}{r^{8}},
$$

enquanto o valor da segunda derivada no ponto de equilíbrio vem a ser

$$
\left.\frac{d^{2} V}{d r^{2}}\right|_{r=r_{0}}=156 \cdot \alpha\left(r_{0}\right)^{-14}-42 \cdot \beta\left(r_{0}\right)^{-8},
$$


que resulta em

$$
\left.\frac{d^{2} V}{d r^{2}}\right|_{r=r_{0}}=36(2 \alpha)^{-4 / 3} \beta^{7 / 3},
$$

que é positivo. Portanto, o ponto $r_{0}$ de fato é um único ponto de equilíbrio estável. O valor do potencial neste ponto é dado por

$$
V\left(r_{0}\right)=-\frac{\beta^{2}}{4 \alpha},
$$

que é também o valor da energia mínima do sistema. Portanto, neste sistema, a energia mecânica pode assumir valores negativos.

Vamos calcular os pontos de retorno do potencial de Lennard-Jones. Os pontos de retorno são raízes da equação $E-V(r)=0$, ou seja,

$$
\frac{\alpha}{r^{12}}-\frac{\beta}{r^{6}}=E
$$

Para $E>0$, esta equação resulta em

$$
r^{12}+r^{6} \frac{\beta}{E}-\frac{\alpha}{E}=0
$$

portanto,

$$
r^{6}=\frac{\beta}{2 E}\left(\sqrt{1+\frac{4 \alpha E}{\beta^{2}}}-1\right) .
$$

A solução negativa implicaria em $r^{6}$ negativo, o que não é uma solução aceitável. Finalmente, temos

$$
r_{-} \equiv \sqrt{\left(\frac{\beta}{2 E}\right)^{1 / 3}\left(\sqrt{1+\frac{4 \alpha E}{\beta^{2}}}-1\right)^{1 / 3}} .
$$

Esta é uma única raiz positiva, portanto, um único ponto de retorno. Assim, para energias positivas, temos apenas um ponto de retorno, que é o ponto de maior aproximação entre os dois átomos. Na figura 3, este caso corresponde ao sistema com energia $E_{3}$, por exemplo.

Se a energia é negativa, temos a equação

$$
r^{12}-r^{6} \frac{\beta}{|E|}+\frac{\alpha}{|E|}=0
$$

ou seja,

$$
r^{6}=\frac{1}{2} \frac{\beta}{|E|}\left(1 \pm \sqrt{1-\frac{4 \alpha}{\beta^{2}}|E|}\right) .
$$

Desde que $E>-\beta^{2} / 4 \alpha$, resultado que já conhecemos, não teremos problemas com raízes imaginárias ou raízes negativas dessas soluções. Ainda, temos que

$$
r_{ \pm} \equiv \sqrt{\left(\frac{\beta}{2|E|}\right)^{1 / 3}\left(1 \pm \sqrt{1-\frac{4 \alpha}{\beta^{2}}|E|}\right)^{1 / 3}}
$$

são os pontos de retorno do sistema. Estes pontos estão ilustrados para o exemplo específico da figura 3, nas interseções entre as retas $E_{1}$ e $E_{2}$ com o potencial $V$.

Como $r_{ \pm}$são pontos de retorno para $E<0$, ainda que a dinâmica temporal seja muito complexa para ser calculada pela quadratura, sabemos que os átomos adquirem um movimento radial oscilatório entre $r_{-}$e $r_{+}$ para cada valor possível de energia. Muitas vezes, como neste caso, o conhecimento dos pontos de retorno é mais significativo que o conhecimento da função horária em si. 
Quando um sistema possui um regime de movimento oscilatório entre dois pontos de retorno, dizemos que o sistema se encontra em um estado ligado. No caso das moléculas diatômicas, os estados ligados são aqueles de energia negativa. Os estados de energia positiva são denominados estados de espalhamento, pois após alcancar a distância $r_{-}$em (??), os átomos entrarão em movimento de escape entre eles, até se tornarem partículas livres.

Um ponto limite para os estados de movimento é o de energia nula. Neste caso, temos também um único ponto de retorno, dado por

$$
r_{-}=\left(\frac{\alpha}{\beta}\right)^{1 / 6}
$$

Veremos, que, no caso do átomo de hidrogênio, considerações similares podem ser feitas. 